\title{
COMBINED SYSTEM FOR DEGASSING WORKING FLUID AND PUMP CHARGING
}

\author{
V. V. Abramov, A. A. Mikhailov, \\ V.G.Shram, A. N. Sokol'nikov, and V.I. Afanasov
}

UDC 621.689

\begin{abstract}
The article reveals the principle of operation of a combined system for degassing working fluid and pump charging by means of a hydraulic accumulator for hydraulic systems with working bodies in the form of hydraulic cylinders. It is concluded that the working fluid can be degassed without affecting pump operation. Due to the absence of a mechanical drive and throttling, the system allows more efficient use of drive energy.
\end{abstract}

Keywords: hydraulics, hydraulic accumulator, liquid, pressure, vacuum, degassing, pump charging.

Many modern self-propelled vehicles of various technological purposes are equipped with hydraulic driven propulsion units or operational equipment. Currently, there is a tendency to increase the nominal pressure and power of hydraulic actuators. At the same time, new increased demands are placed on the performance of hydraulic actuators and the service life of the working fluid. The main factors affecting the performance of hydraulic actuators are: temperature, contamination of the working fluid, the presence of dissolved and undissolved gas in the working fluid.

The presence of gas in the working fluid is the main cause of oxidation of the working fluid, the formation of acids, corrosion of hydraulic equipment, deterioration of pump suction capacity, cavitation phenomena and the diesel effect. All these factors intensify aging of the working fluid and, as a consequence, lead to frequent replacement of the working fluid, decreasing the efficiency and productivity of hydraulic machines.

The known physical methods for degassing working fluid include: incubation in hydraulic tanks, mechanical destruction of bubbles, filtration, evacuation, cavitation, centrifugation, and others. Each of these methods has its own drawbacks. The most acceptable in terms of efficiency and economic feasibility is the method of evacuation with the installation of the drain pipe near the level of the working fluid in the hydraulic tank.

We propose a method for significantly reducing negative effects of the gas phase (contained in the working fluid) by applying a closed hydraulic system with an accumulator feed system in combination with a degassing device.

A closed circulation hydraulic system with accumulator feed and a degassing device has several advantages. During the operation of such hydraulic systems in cold climates, the following are provided: improved pump suction conditions (by creating an overpressure at the pump inlet by means of a charging accumulator); reduced amount of the gas phase in the working fluid; increased feed rate of the main pump.

A possible arrangement of the hydraulic system with working bodies in the form of hydraulic cylinders and an accumulator charging system is given in Fig. 1.

The charging system is based on a hydraulic accumulator 1 , control valve 2 , hydraulic distributor 3 , safety valve 4 , throttle 5 .

Gubkin Russian State University of Oil and Gas (National Research University), Moscow, Russia; e-mail: VAbramov@sfu-kras.ru.

Translated from Khimicheskoe i Neftegazovoe Mashinostroenie, Vol. 55, No. 3, pp. 16-18, March, 2019. 


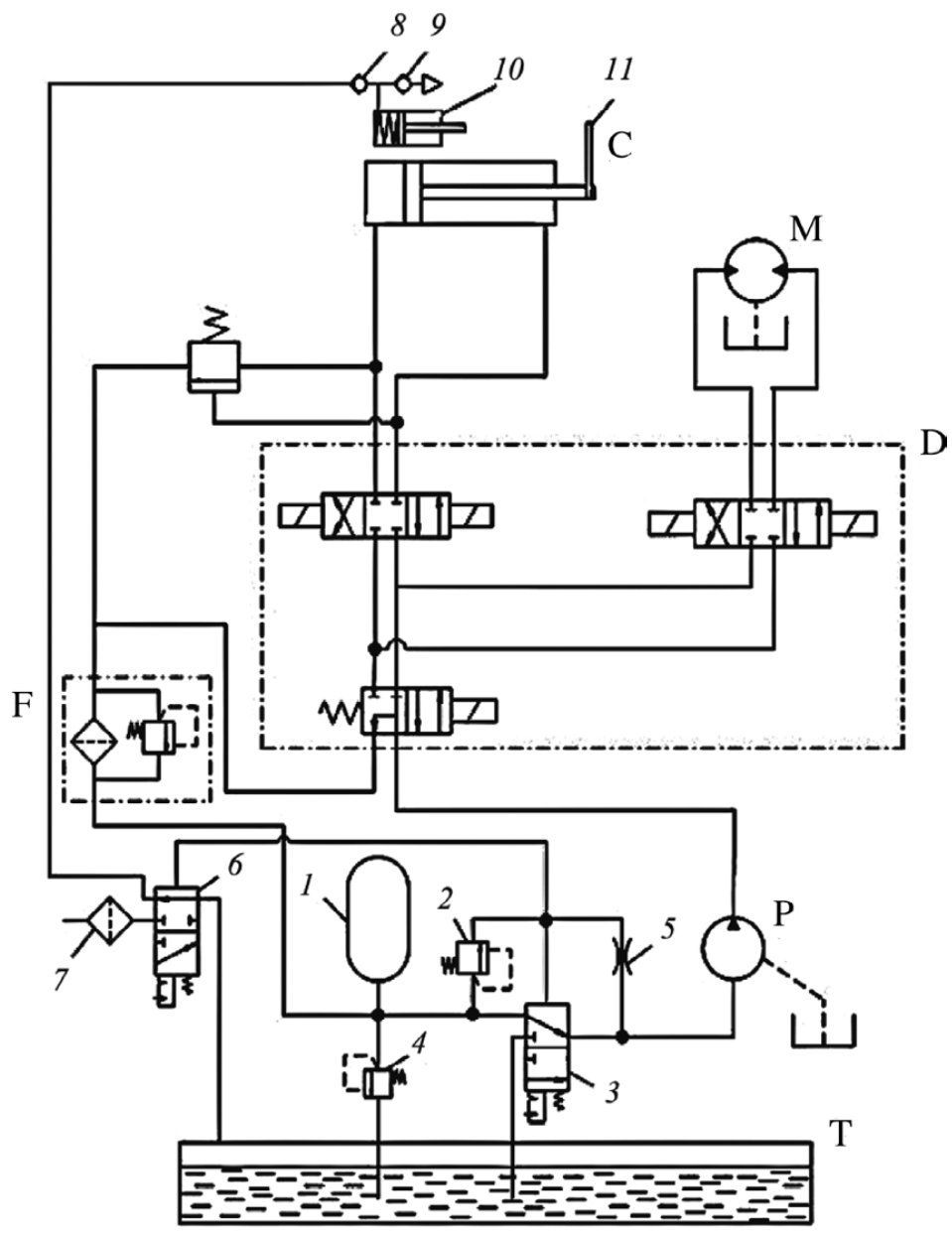

Fig. 1. A closed circulation hydraulic system with accumulator pump charging and a device for degassing the working fluid: $\mathrm{T}-$ hydraulic tank; M - hydraulic motor; C - hydraulic cylinder; P - pump; D - distributor; F - filter.

The degassing device consists of a pneumatic distributor 6 , an air filter 7 , check valves 8 and 9 , a pneumatic cylinder 10 (as a vacuum pump) and a stand 11 .

The hydraulic accumulator is installed on the drain line connected to the suction line of the pump through the valve 3 (with two fixed positions). At one end of the slide valve there is a control cavity connected through the throttle 5 to the pump suction line, as well as to the output of the control valve 2 and to the pneumatic control chamber 6. At the other end of the slide valve, a spring at a certain pressure in the suction line shifts the slide valve into position corresponding to the charging of the accumulator. In this case, the fluid from the control cavity is forced through the throttle into the suction line of the pump. Simultaneously, under the action of the spring, the valve of the pneumatic distributor moves to the position in which the hydraulic tank communicates with the atmosphere through the air filter. At the same time, vacuum pressure is created in the piston cavity of the pneumatic cylinder due to the alternate opening/closing of the check valves 8,9 and the reciprocating movement of the pneumatic cylinder rod, after which the pneumatic cylinder rod remains in the retracted position. In this case, degassing does not take place.

Charging and degassing are carried out cyclically. At pressures below the set value, the discharge line after the accumulator is blocked by the hydraulic distributor, while the accumulator is charged and the working 
fluid is drawn in from the hydraulic tank. When the pressure in the hydroaccumulator increases to a value corresponding to its sufficient charge, the control valve 2 switches the hydraulic valve 3 and the pneumatic valve 6 . With this position of the pneumatic valve, the gas chamber of the tank is connected to the pneumatic cylinder 10. During operation of the machine cylinder piston rod, the reciprocating movement through the rack 11 is transmitted to the pneumatic cylinder, while the working fluid is evacuated in the hydraulic tank. The result is an intense separation of the gas phase from the working fluid. At the same time, the slide valve moves to the position corresponding to the connection of the drain and suction lines of the hydraulic system. Reverse flow of fluid into the hydraulic tank from the suction line is prevented directly by the valve. Leaks in the system and outflow fluctuations are compensated by a hydraulic accumulator. Excessive pressure increase in the charging system is limited by the safety valve 4 . The working fluid (mixture of oil and air effectively deaerated by the degassing device) enters the hydraulic tank only through the drainage lines of hydraulic machines and hydraulic equipment.

Due to the cyclical nature of the system (recharge and degassing), degassing does not affect the process of suction of the working fluid by the pump, which will allow for the application of a higher negative pressure and thus increase the efficiency of degassing.

The working volume $\left(\mathrm{m}^{3}\right)$ of the hydraulic accumulator of the charging system is determined from the expression:

$$
V_{\mathrm{ac}}=\Delta V_{\mathrm{hc} \cdot \max }+\sum V_{\mathrm{lk}},
$$

where

$\Delta V_{\text {hc.max }}$ is the maximum change in the volume of the cavities of the hydraulic cylinders in the cycle;

$\sum V_{\mathrm{lk}}$ is the total leakage of pumps and motors through drainage lines during the cycle.

Thus, for a single operation of each hydraulic cylinder during the operating cycle, the minimum working volume of the accumulator will be

$$
V_{\mathrm{ac}}=\sum_{i=1}^{n} \frac{\pi d_{i}^{2}}{4} l_{i}+\sum V_{\mathrm{lk}}
$$

where $n$ is the number of working cylinders during the full cycle; $i$ is the number of the hydraulic cylinder operating in the cycle; $d$ is the diameter of the hydraulic cylinder; $l$ is the stroke of the hydraulic cylinder.

Charging time of the hydraulic accumulator $t_{\mathrm{c}}(\mathrm{s})$ at the neutral position of the slide valve is

$$
t_{\mathrm{c}}=V_{\mathrm{ac}} /\left(Q_{\mathrm{p}}-Q_{\mathrm{lk}}\right)
$$

where

$V_{\text {ac }}$ is the working volume of the accumulator, $\mathrm{m}^{3}$;

$Q_{\mathrm{p}}$ is the volumetric pump flow, $\mathrm{m}^{3} / \mathrm{s}$;

$Q_{\mathrm{lk}}$ is the leakage flow rate in hydraulic equipment, $\mathrm{m}^{3} / \mathrm{s}$. 
If the pressure in the charging system is lower than the allowable, the system automatically switches to charging the accumulator, while the function of the fluid volume $\left(\mathrm{m}^{3}\right)$ in the accumulator (during charging and subsequent operation, taking into account leaks in the pump and during idling of the hydraulic system) will have the form

$$
V_{\mathrm{ac} . \text { idl }}(t)=V_{\mathrm{a}}(t)\left(0 \leq t \leq t_{\mathrm{c}}\right)+V_{\mathrm{a}}\left(t_{\mathrm{c}}\right)\left(t>t_{\mathrm{c}}\right)-V_{\mathrm{lk}}(t)\left(0 \leq t \leq t_{\mathrm{cy}}\right)
$$

where $V_{\mathrm{a}}(t)$ is the volume of liquid that entered the accumulator from the pump during time $t ; V_{\mathrm{lk}}(t)$ is the volume of leaks in the hydraulic system during time $t ; t_{\mathrm{cy}}$ is the time of the operating cycle of the hydraulic drive.

For complex hydraulic operating cycles, the required working volume of the hydraulic accumulator can be determined using the function of changing the volume of fluid in the accumulator during the operating cycle $\left(\mathrm{m}^{3}\right)$ :

$$
V_{\text {ac.op.cy }}(t)=V_{\text {ac.idl }}(t)-Q_{\mathrm{p}} \sum_{i=1}^{n} \sum_{j=1}^{m}\left(1-c_{i, j}\right)\left(\left(t-a_{i, j}\right)\left(a_{i, j}<t \leq b_{i, j}\right)+\left(b_{i, j}-a_{i, j}\right)\left(b_{i, j}<t \leq t_{\mathrm{cy}}\right)\right),
$$

where $i$ is the number of the hydraulic cylinder; $j$ is the sequence number of inclusion of the $i$-th hydraulic cylinder in the cycle; $c_{i, j}=\psi_{i}^{-1}$ is the parameter of the direct stroke of the hydraulic cylinder; $c_{i, j}=\psi_{i}$ is the parameter of the return stroke of the hydraulic cylinder $\left(\psi_{\mathrm{i}}=S_{\mathrm{p}} / S_{\mathrm{p} . \mathrm{r}}\right.$ is the ratio of the areas of the piston in the piston and rod cavities); $a_{i, j}, b_{i, j}$ represent the time of the beginning and end of the movement of the rod the $i$-th hydraulic cylinder during the $j$-th activation, respectively.

Equation (4) is obtained under the assumption that the beginning of the working cycle is synchronized with the beginning of the accumulator charging period. In the general case, it is possible to unsynchronize the time of the accumulator charging cycle and the time of the working cycle, thus obtaining an arbitrary picture of the joint operation of the charging and hydraulic drive systems. Also, when modeling, it is necessary to consider that the maximum accumulator volume is structurally limited and cannot be exceeded.

For hydraulic systems, in which hydraulic cylinders with one-sided rods are used as working bodies, it is necessary to further increase the working volume of the accumulator and not fully charge it, but taking into account a possible increase in the discharge flow during the return stroke of the hydraulic cylinders (according to the hydraulic drive operating cycle). If the charging pressure of the charging system is set correctly and the accumulator volume is sufficiently uncharged, the increase in pressure in the charging system above the allowable one can be completely eliminated, which will prevent energy loss when draining the liquid through the safety valve of the charging system.

Hydraulic accumulator systems are preferably used in high-pressure hydraulic systems, where (compared to low-pressure hydraulic systems) for the same power, the pump feed is less, and correspondingly a hydraulic accumulator of a smaller volume is required.

While the working fluid enters the pump from the charging system, and not from the hydraulic tank, evacuation occurs, allowing to increase the Stack effect in the hydraulic tank without reducing the efficiency of pump suction. When using such a system, the processes of charging and degassing are carried out automatically, without operator intervention. In addition, a mechanical drive and throttling are not required for the operation of the charging system, allowing more efficient use of drive energy. 


\section{REFERENCES}

1. A. A. Mikhailov, S. V. Kaverzin, A. S. Kaverzina, and V. V. Abramov, "The influence of the gas phase in the working fluid on the performance of a hydraulic actuator," Mashinostroenie: Coll. Sci. Articles, Exec. ed. E. G. Sinenko, IPK SFU, Krasnoyarsk, 58-63 (2009).

2. V. V. Abramov, "Closed hydraulic system with an accumulator charging system," Abstracts Int. Sci. Technol. Conf. "Hydraulic machines, hydraulic drives and hydropneumatic automation. The current state and development prospects” [in Russian], SPb (2008), pp. 176-177.

3. Pat. RF No. 2044841, “A hydraulic system for mobile equipment” [in Russian], E. I. Avdeev and N. S. Revnyakov, Publ. September 27, 1995.

4. Pat. RF No. 85920, “A hydraulic system for mobile equipment” [in Russian], A. A. Mikhailov, V. V. Abramov, and S. V. Kaverzin, Bull. No. 23 (2009).

5. Pat. RF No. 2435909, "A hydraulic system for mobile equipment” [in Russian], V. V. Abramov and A. A. Mikhailov, Bull. No. 34 (2011). 